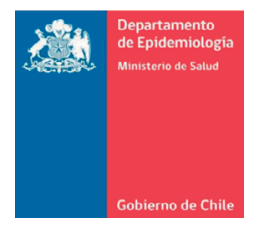

\title{
Informe: \\ Situación epidemiológica de las infecciones de transmisión sexual en Chile, 2017
}

Report: Epidemiological situation of sexually transmitted infections in Chile, 2017

Sexually transmitted infections (STIs) are one of the main causes of acute illness, infertility, long-term disability and death in the world ${ }^{1}$. This report presents the preliminary epidemiological situation of STIs up to the year 2017 in Chile. Syphilis is the STI with the highest reporting rate, followed by HIV infection. In general terms, all STIs present a relative stabilization of their rates in the 2014 and 2015 periods, except for gonorrhea that shows an increase in these years, which focuses on the group of 15 to 24 years. In 2017, syphilis and HIV infection showed an increase in their rates in relation to 2016. In all these STIs the rate of men exceeds that of women and the most affected age group is 15 to 39 years. According to geographical distribution, the regions of Arica-Parinacota to Antofagasta, Metropolitana, Valparaíso, Los Lagos and Aysén, present the greatest risks.

Keywords: Sexually transmitted infections; Chilean epidemiology.

Palabras clave: Infecciones de transmisión sexual; epidemiología chilena.

\section{Antecedentes}

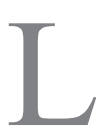

as infecciones de transmisión sexual (ITS) son una de las principales causas de enfermedad aguda, infertilidad, discapacidad a largo plazo y muerte en el mundo ${ }^{1}$. Su principal forma de transmisión es por relaciones sexuales no protegidas y ocasionalmente por transmisión madre a hijo ${ }^{2}$.

Las ITS imponen a los países, tanto de recursos limitados como a los desarrollados, una gran carga de morbilidad y mortalidad, ya sea de forma directa, por la repercusión que tienen en la calidad de vida, la salud reproductiva y la salud del niño, o bien indirecta, por su función facilitadora de la trasmisión sexual del VIH y su impacto en las economías nacionales e individuales ${ }^{2}$.

Nuevas investigaciones indican que se da una interacción importante entre la infección muy temprana por VIH y otras ITS. Esa interacción podría explicar $40 \%$ o más de los casos de transmisión del $\mathrm{VIH}^{2}$.

Existe una mayor carga de morbilidad, particularmente en los países en desarrollo, pero en los países industrializados se prevé un aumento de la carga de morbilidad debido a la prevalencia de infecciones víricas incurables, a las tendencias en el comportamiento sexual y al incremento de los viajes ${ }^{2}$.

En Chile, el Decreto Supremo 158/2004 establece que la sífilis (todas sus formas clínicas), la gonorrea, la hepatitis B y la infección por VIH son infecciones de declaración obligatoria universal. Además, Neisseria gonorrhoeae, el virus de la hepatitis B y el VIH son agentes de vigilancia de laboratorio ${ }^{3}$.

El propósito de la vigilancia epidemiológica es entregar información que permita orientar la toma de decisiones y la planificación de estrategias de prevención y control. En este sentido, el objetivo de este informe es describir la tendencia y el comportamiento de los casos de ITS bajo vigilancia en Chile hasta el año 2017.

\section{Método}

El presente informe es un análisis de tipo descriptivo de los casos de infección por VIH, gonorrea, hepatitis B y sífilis notificados en Chile hasta el año 2017. Para su elaboración se utilizó la información proveniente de bases de datos secundarias:

- Para gonorrea (código CIE-10: A54), hepatitis B (código CIE-10: B16; B18.0-B18.1) y sífilis (código CIE10: A50-A53.9), se trabajó con la base de datos según CIE-10 de enfermedades de notificación obligatoria (ENO), dependiente del Departamento de Epidemiología del Ministerio de Salud de Chile (MINSAL) y del Departamento de Estadísticas e Información en Salud (DEIS). Esta información se encuentra validada hasta el año 2015, la del año 2015 hasta 2017 corresponde a información provisoria.

- Para VIH, se trabajó con:

a. Datos proporcionados por el registro del sistema de notificación de casos en línea, los que son incorporados una vez que la persona ingresa a control y es clasificada según etapa de la infección (VIH o Sida) o bien cuando han fallecido.

b. Datos provenientes de los certificados de defunción validados hasta el año 2015 por el DEIS del Ministerio de Salud de Chile.
(C)Departamento de Epidemiología. http://epi.minsal.cl

División de Planificación Sanitaria Ministerio de Salud de Chile.

Correspondencia a: Karen Cáceres Burton karen.caceres@minsal.cl 
c. Estimaciones realizadas con software Spectrum, proporcionado por ONUSIDA a nivel mundial*.

d. Informe sobre casos confirmados por el Instituto de Salud Pública (ISP) al año 2015.

Las definiciones de caso de cada infección, se encuentran descritas en sus respectivas circulares, disponibles en: http://epi.minsal.cl/

Se presentan indicadores descriptivos para el análisis por tiempo, lugar, persona (sexo, edad, clasificación de la infección por CIE-10) y determinantes sociales (nacionalidad y pertenencia a pueblos originarios). Se calcularon porcentajes, medianas y tasas; estas últimas se amplificaron por cien mil habitantes (habs.) utilizando las proyecciones de población del Instituto Nacional de Estadística (INE) del año 2012. Se usaron tablas para presentar los resultados utilizando el software Microsoft Excel.

Con relación a la Ley $\mathrm{N}^{\circ} 20.584$ que "Regula los derechos y deberes que tienen las personas en relación con acciones vinculadas a su atención en salud", este informe no vulnera la mencionada ley, debido a que utiliza datos del Ministerio de Salud, los que se recogen dando cumplimiento a su rol según las siguientes leyes previas: DFL No $1 / 2005$ del MINSAL y Ley $N^{\circ} 19.628$ sobre datos sensibles.

\section{Limitaciones}

En los resultados de la vigilancia de infección por VIH, es importante señalar que el informe sólo presenta tasas de incidencia de nuevos diagnósticos notificados, por lo que a partir de esta fuente de información no es posible conocer la tasa de incidencia de infecciones por VIH, ni el número de nuevas personas contagiadas.

Las notificaciones se realizan de acuerdo al año en que las personas fueron etapificadas, lo que no necesariamente es coincidente con el año en que el ISP confirma los casos. Esto debido a que existe una latencia de tiempo desde que una persona es confirmada hasta que accede al control médico, momento en el cual es etapificado, en base a exámenes de laboratorio (recuento de LTCD4 y carga viral) y etapa clínica (de acuerdo a definición de caso).

Para gonorrea, hepatitis B y sífilis, se debe considerar que se trabaja a partir de las notificaciones obligatoriasuniversales a nivel nacional. No obstante, podría existir sub-notificación de casos, lo que limita conocer la realidad de la infección; además, existen algunas deficiencias en

* Detalles de la metodología de estimaciones Spectrum se encuentran disponible en:

http://www.unaids.org/es/resources/presscentre/featurestories/ 2017/july/20170710_data

http://aidsinfo.unaids.org/documents/estimates methods 2017.pdf la calidad en ciertas variables de los datos ingresados (existen registros incompletos que no permiten hacer análisis más profundos). Para disminuir la sub-notificación y mejorar la calidad de la información, las Secretarias Regionales Ministeriales (SEREMI) de Salud trabajan en el refuerzo constante de la vigilancia en la red pública y privada de salud.

\section{Situación epidemiológica según infección de transmisión sexual}

\section{Infección por $\mathrm{VIH}$}

Según los cálculos realizados por software Spectrum, al año 2017 se estima que en Chile se infectan 5.000 casos anualmente, con una prevalencia en población de 15 a 49 años de $0,5 \%$, con 65.000 mil casos de infección por VIH.

Según informa el ISP (información que corresponde a $100 \%$ de los nuevos diagnósticos de infección por VIH conocidos confirmados por el sistema de salud), en el período 2010-2015 se confirmaron 21.856 nuevos diagnósticos (mediana de 4.004 casos confirmados anualmente). La tasa anual de casos confirmados experimentó una tendencia al alza desde el año 2010 con una tasa de 21,4 casos por 100.000 habs. $\geq 13$ años, hasta el 2015 con una tasa de 28,9 casos por 100.000 habs. $\geq 13$ años. Según distribución regional (en los mayores de 13 años), las regiones de Arica- Parinacota, Tarapacá y Metropolitana registraron las tasas más altas para cada año del período en estudio. Se observa, además, un aumento continuo en las tasas de Coquimbo, Valparaíso y Magallanes desde el año 2010 hasta el 2015 (informe disponible en: http://www. ispch.cl/sites/default/files/BoletinVIH-27022017B.pdf).

Según la información de la vigilancia epidemiológica, se evidenció que a partir del año 2011 se presentó una relativa estabilización de las tasas de notificación entre 14 y 15 por cien mil habs.; ésta incrementó hasta 17,9 por cien mil habs. el año 2017. El 66\% de los casos se notificó en etapa VIH (etapas tempranas) (Tabla 1).

El principal grupo poblacional afectado fue el de adultos entre 20 y 39 años. Específicamente el grupo de 25 a 29 años muestra la tasa más alta el año 2017 (50,6 por cien mil habs.) El grupo de adolescentes (15 a 19 años) presentó tasas estables hasta el año 2015; sin embargo, el año 2016 y 2017 (tasa de 9,2 y 9,1 por cien mil habs. respectivamente), mostró un aumento de $20 \%$ en relación al año 2015. El grupo de adultos mayores (60 y más años) mostró estabilización de sus tasas en los últimos dos años (Tabla 2).

En el año 2017 existió una relación de 5,9 hombres por cada mujer (Tabla 3), observándose este año un aumento tanto en la tasa de hombres como de mujeres con respecto a los años anteriores. La principal vía de transmisión fue la sexual, representa $99 \%$ en el quinquenio 2013-2017, 


\begin{tabular}{|c|c|c|c|c|c|c|c|c|c|c|c|c|c|c|c|c|c|c|}
\hline \multirow{3}{*}{ Año } & \multicolumn{6}{|c|}{ SIDA } & \multicolumn{6}{|c|}{ VIH } & \multicolumn{6}{|c|}{ VIH/SIDA } \\
\hline & \multicolumn{2}{|c|}{ Hombres } & \multicolumn{2}{|c|}{ Mujeres } & \multicolumn{2}{|c|}{ Total } & \multicolumn{2}{|c|}{ Hombres } & \multicolumn{2}{|c|}{ Mujeres } & \multicolumn{2}{|c|}{ Total } & \multicolumn{2}{|c|}{ Hombres } & \multicolumn{2}{|c|}{ Mujeres } & \multicolumn{2}{|c|}{ Total } \\
\hline & $\mathbf{n}$ & Tasa & $\mathbf{n}$ & Tasa & $\mathbf{n}$ & Tasa & $\mathbf{n}$ & Tasa & $\mathbf{n}$ & Tasa & $\mathbf{n}$ & Tasa & $\mathbf{n}$ & Tasa & $\mathbf{n}$ & Tasa & $\mathbf{n}$ & Tasa \\
\hline 1984 & 6 & 0,1 & 0 & 0,0 & 6 & 0,1 & 0 & 0,0 & 0 & 0,0 & 0 & 0,0 & 6 & 0,0 & 0 & 0,0 & 6 & 0,1 \\
\hline 1985 & 9 & 0,2 & 1 & 0,0 & 10 & 0,1 & 1 & 0,0 & 0 & 0,0 & 1 & 0,0 & 10 & 0,2 & 1 & 0,0 & 11 & 0,1 \\
\hline 1986 & 19 & 0,3 & 1 & 0,0 & 20 & 0,2 & 6 & 0,1 & 0 & 0,0 & 6 & 0,0 & 25 & 0,4 & 1 & 0,0 & 26 & 0,2 \\
\hline 1987 & 50 & 0,8 & 1 & 0,0 & 51 & 0,4 & 22 & 0,4 & 3 & 0,0 & 25 & 0,2 & 72 & 1,2 & 4 & 0,1 & 76 & 0,6 \\
\hline 1988 & 73 & 1,2 & 15 & 0,2 & 88 & 0,7 & 31 & 0,5 & 9 & 0,1 & 40 & 0,3 & 104 & 1,7 & 24 & 0,4 & 128 & 1,0 \\
\hline 1989 & 128 & 2,0 & 11 & 0,2 & 139 & 1,1 & 64 & 1,0 & 7 & 0,1 & 71 & 0,5 & 192 & 3,0 & 18 & 0,3 & 210 & 1,6 \\
\hline 1990 & 238 & 3,7 & 13 & 0,2 & 251 & 1,9 & 95 & 1,5 & 14 & 0,2 & 109 & 0,8 & 333 & 5,1 & 27 & 0,4 & 360 & 2,7 \\
\hline 1991 & 310 & 4,7 & 36 & 0,5 & 346 & 2,6 & 157 & 2,4 & 23 & 0,3 & 180 & 1,3 & 467 & 7,0 & 59 & 0,9 & 526 & 3,9 \\
\hline 1992 & 328 & 4,9 & 35 & 0,5 & 363 & 2,7 & 166 & 2,5 & 26 & 0,4 & 192 & 1,4 & 494 & 7,3 & 61 & 0,9 & 555 & 4,1 \\
\hline 1993 & 326 & 4,7 & 33 & 0,5 & 359 & 2,6 & 210 & 3,1 & 31 & 0,4 & 241 & 1,7 & 536 & 7,8 & 64 & 0,9 & 600 & 4,3 \\
\hline 1994 & 366 & 5,2 & 45 & 0,6 & 411 & 2,9 & 207 & 3,0 & 41 & 0,6 & 248 & 1,8 & 573 & 8,2 & 86 & 1,2 & 659 & 4,7 \\
\hline 1995 & 370 & 5,2 & 40 & 0,5 & 410 & 2,8 & 261 & 3,7 & 43 & 0,6 & 304 & 2,1 & 631 & 8,9 & 83 & 1,1 & 714 & 5,0 \\
\hline 1996 & 404 & 5,6 & 68 & 0,9 & 472 & 3,2 & 347 & 4,8 & 87 & 1,2 & 434 & 3,0 & 751 & 10,4 & 155 & 2,1 & 906 & 6,2 \\
\hline 1997 & 412 & 5,6 & 46 & 0,6 & 458 & 3,1 & 392 & 5,4 & 92 & 1,2 & 484 & 3,3 & 804 & 11,0 & 138 & 1,8 & 942 & 6,4 \\
\hline 1998 & 418 & 5,6 & 59 & 0,8 & 477 & 3,2 & 429 & 5,8 & 99 & 1,3 & 528 & 3,5 & 847 & 11,4 & 158 & 2,1 & 1.005 & 6,7 \\
\hline 1999 & 447 & 5,9 & 80 & 1,0 & 527 & 3,5 & 510 & 6,8 & 117 & 1,5 & 627 & 4,1 & 957 & 12,7 & 197 & 2,6 & 1154 & 7,6 \\
\hline 2000 & 438 & 5,7 & 68 & 0,9 & 506 & 3,3 & 562 & 7,4 & 135 & 1,7 & 697 & 4,5 & 1.000 & 13,1 & 203 & 2,6 & 1.203 & 7,8 \\
\hline 2001 & 466 & 6,0 & 60 & 0,8 & 526 & 3,4 & 557 & 7,2 & 141 & 1,8 & 698 & 4,5 & 1.023 & 13,3 & 201 & 2,6 & 1.224 & 7,9 \\
\hline 2002 & 466 & 6,0 & 58 & 0,7 & 524 & 3,3 & 713 & 9,2 & 171 & 2,2 & 884 & 5,6 & 1.179 & 15,2 & 229 & 2,9 & 1.408 & 9,0 \\
\hline 2003 & 473 & 6,0 & 65 & 0,8 & 538 & 3,4 & 765 & 9,8 & 175 & 2,2 & 940 & 5,9 & 1.238 & 15,8 & 240 & 3,0 & 1.478 & 9,3 \\
\hline 2004 & 458 & 5,8 & 59 & 0,7 & 517 & 3,2 & 706 & 8,9 & 174 & 2,2 & 880 & 5,5 & 1.164 & 14,7 & 233 & 2,9 & 1.397 & 8,7 \\
\hline 2005 & 405 & 5,1 & 84 & 1,0 & 489 & 3,0 & 702 & 8,8 & 169 & 2,1 & 871 & 5,4 & 1.107 & 13,8 & 253 & 3,1 & 1.360 & 8,4 \\
\hline 2006 & 360 & 4,5 & 56 & 0,7 & 416 & 2,5 & 560 & 6,9 & 207 & 2,5 & 767 & 4,7 & 920 & 11,4 & 263 & 3,2 & 1.183 & 7,2 \\
\hline 2007 & 501 & 6,1 & 102 & 1,2 & 603 & 3,7 & 546 & 6,7 & 164 & 2,0 & 710 & 4,3 & 1.047 & 12,8 & 266 & 3,2 & 1.313 & 8,0 \\
\hline 2008 & 582 & 7,0 & 120 & 1,4 & 702 & 4,2 & 667 & 8,1 & 184 & 2,2 & 851 & 5,1 & 1.249 & 15,1 & 304 & 3,6 & 1.553 & 9,3 \\
\hline 2009 & 757 & 9,1 & 111 & 1,3 & 868 & 5,1 & 1.010 & 12,1 & 227 & 2,7 & 1.237 & 7,3 & 1.767 & 21,1 & 338 & 4,0 & 2.105 & 12,5 \\
\hline 2010 & 596 & 7,1 & 106 & 1,2 & 702 & 4,1 & 853 & 10,1 & 192 & 2,2 & 1.045 & 6,1 & 1.449 & 17,2 & 298 & 3,5 & 1.747 & 10,2 \\
\hline 2011 & 875 & 10,2 & 153 & 1,8 & 1.028 & 6,0 & 1.394 & 16,3 & 260 & 3,0 & 1.654 & 9,6 & 2.269 & 26,6 & 413 & 4,7 & 2.682 & 15,5 \\
\hline 2012 & 796 & 9,2 & 138 & 1,6 & 934 & 5,4 & 1.243 & 14,4 & 264 & 3,0 & 1.507 & 8,6 & 2.039 & 23,6 & 402 & 4,6 & 2.441 & 14,0 \\
\hline 2013 & 827 & 9,5 & 148 & 1,7 & 975 & 5,5 & 1.323 & 15,2 & 275 & 3,1 & 1.598 & 9,1 & 2.150 & 24,6 & 423 & 4,8 & 2.573 & 14,6 \\
\hline 2014 & 856 & 9,7 & 162 & 1,8 & 1.018 & 5,7 & 1.462 & 16,6 & 268 & 3,0 & 1.730 & 9,7 & 2.318 & 26,3 & 430 & 4,8 & 2.748 & 15,4 \\
\hline $2015^{*}$ & 904 & 10,1 & 163 & 1,8 & 1.067 & 5,9 & 1.489 & 16,7 & 253 & 2,8 & 1.742 & 9,7 & 2.393 & 26,9 & 416 & 4,6 & 2.809 & 15,6 \\
\hline $2016^{*}$ & 961 & 10,7 & 148 & 1,6 & 1.109 & 6,1 & 1.618 & 18,0 & 266 & 2,9 & 1.884 & 10,4 & 2.579 & 28,6 & 414 & 4,5 & 2.993 & 16,5 \\
\hline $2017^{*}$ & 948 & 10,4 & 176 & 1,9 & 1.124 & 6,1 & 1.869 & 20,6 & 298 & 3,2 & 2.167 & 11,8 & 2.817 & 31,0 & 474 & 5,1 & 3.291 & 17,9 \\
\hline Total & 15.573 & & 2.461 & & 18.034 & & 20.937 & & 4.415 & & 25.352 & & 36.510 & & 6.876 & & 43.386 & \\
\hline
\end{tabular}




\begin{tabular}{|c|c|c|c|c|c|c|c|c|c|c|}
\hline \multirow[t]{2}{*}{ Grupos de edad } & \multicolumn{2}{|c|}{2013} & \multicolumn{2}{|c|}{2014} & \multicolumn{2}{|c|}{2015} & \multicolumn{2}{|c|}{ 2016* } & \multicolumn{2}{|c|}{$2017^{*}$} \\
\hline & Casos & Tasa & Casos & Tasa & Casos & Tasa & Casos & Tasa & Casos & Tasa \\
\hline 0 - 4 años & 3 & 0,2 & 4 & 0,3 & 11 & 0,9 & 8 & 0,6 & 19 & 1,5 \\
\hline $5-9$ años & 1 & 0,1 & 0 & 0,0 & 3 & 0,2 & 3 & 0,2 & 3 & 0,2 \\
\hline $10-14$ años & 1 & 0,1 & 3 & 0,2 & 1 & 0,1 & 3 & 0,3 & 0 & 0,0 \\
\hline $15-19$ años & 106 & 7,7 & 96 & 7,1 & 100 & 7,6 & 120 & 9,2 & 116 & 9,1 \\
\hline 20 - 24 años & 534 & 35,8 & 540 & 36,5 & 564 & 38,6 & 545 & 37,9 & 590 & 41,7 \\
\hline $25-29$ años & 508 & 35,2 & 565 & 38,4 & 572 & 38,2 & 687 & 45,3 & 772 & 50,6 \\
\hline $30-34$ años & 428 & 33,3 & 441 & 33,4 & 444 & 32,7 & 517 & 37,0 & 590 & 41,1 \\
\hline 35 - 39 años & 259 & 20,9 & 301 & 24,3 & 326 & 26,2 & 345 & 27,5 & 393 & 30,8 \\
\hline 40 - 44 años & 241 & 19,3 & 260 & 20,9 & 252 & 20,3 & 248 & 19,9 & 292 & 23,5 \\
\hline 45 - 49 años & 210 & 16,6 & 230 & 18,2 & 213 & 16,9 & 185 & 14,8 & 191 & 15,3 \\
\hline 50 - 54 años & 124 & 10,5 & 147 & 12,2 & 134 & 11,0 & 141 & 11,4 & 155 & 12,4 \\
\hline 55 - 59 años & 80 & 8,3 & 67 & 6,6 & 95 & 9,0 & 96 & 8,8 & 80 & 7,1 \\
\hline $60-64$ años & 41 & 5,4 & 53 & 6,7 & 50 & 6,1 & 57 & 6,6 & 46 & 5,1 \\
\hline 65 - 69 años & 22 & 3,7 & 23 & 3,7 & 23 & 3,6 & 23 & 3,5 & 29 & 4,2 \\
\hline 70 - 74 años & 9 & 2,0 & 11 & 2,4 & 11 & 2,3 & 10 & 2,0 & 10 & 1,9 \\
\hline 75 - 79 años & 3 & 0,9 & 6 & 1,8 & 9 & 2,6 & 4 & 1,1 & 4 & 1,1 \\
\hline 80 y más años & 1 & 0,3 & 1 & 0,3 & 1 & 0,3 & 1 & 0,2 & 1 & 0,2 \\
\hline Chile & 2.571 & 14,6 & 2.748 & 15,4 & 2.809 & 15,6 & 2.993 & 16,5 & 3.291 & 17,9 \\
\hline
\end{tabular}

Tabla 3. Casos y tasas de notificaciones por VIH según sexo. Chile 2013-2017*

\begin{tabular}{|ccccccc}
\hline Año & Casos & Hombres & Mujeres & Relación Hombre:Mujer \\
& 2.150 & 24,6 & 423 & Tasa & 4,8 & 5,1 \\
\hline 2013 & 2.318 & 26,3 & 430 & 4,8 & 5,4 \\
\hline 2014 & 2.393 & 26,9 & 416 & 4,6 & 5,8 \\
\hline $2016^{*}$ & 2.579 & 28,6 & 414 & 4,5 & 6,2 \\
\hline $2017^{*}$ & 2.817 & 31,0 & 474 & 5,1 & 5,9 \\
\hline
\end{tabular}

Fuente: Formulario notificación de VIH/Sida, Depto Eidamiología, MINSAL. Tasa por cien mil habs. *Datos en validación.

siendo la principal práctica de riesgo declarada la relación sexual de hombres con otros hombres, lo que concentra $66 \%$ de los casos en este mismo quinquenio.

En el último quinquenio, 11,6\% del total de casos notificados correspondió a personas extranjeras, siendo $85 \%$ de sexo masculino. El año 2017 el porcentaje de extranjeros notificados representó $19 \%$ del total de casos. E1 3,6\% de total de casos notificados declaró pertenecer a algún pueblo originario en este último quinquenio.
A nivel territorial, en el mismo período, las mayores tasas se concentran en el norte y centro del país. El año 2017, la Región de Arica-Parinacota presenta las tasas más altas del país (50,6 por cien mil habs.) superando en 2,8 veces la tasa nacional. La Región de Tarapacá mostró una tasa de 24,7 por cien mil habs., ocupando el segundo lugar. Le siguen las regiones Metropolitana y Atacama; esta última triplica su tasa el año 2016 en relación al 2015. Sigue la Región de Antofagasta con una tasa de 19,2 por 


\begin{tabular}{|c|c|c|c|c|c|c|c|c|c|c|}
\hline \multirow[t]{3}{*}{ Región } & \multicolumn{2}{|c|}{2013} & \multicolumn{2}{|c|}{2014} & \multicolumn{2}{|c|}{2015} & \multicolumn{2}{|c|}{$2016^{*}$} & \multicolumn{2}{|c|}{$2017^{*}$} \\
\hline & Casos & Tasa & Casos & Tasa & Casos & Tasa & Casos & Tasa & Casos & Tasa \\
\hline & \multicolumn{10}{|c|}{ VIH/SIDA } \\
\hline Arica y Parinacota & 55 & 23,8 & 69 & 29,4 & 107 & 44,7 & 101 & 41,5 & 125 & 50,6 \\
\hline Tarapacá & 62 & 19,3 & 69 & 21,0 & 66 & 19,6 & 128 & 37,1 & 87 & 24,7 \\
\hline Antofagasta & 113 & 18,7 & 66 & 10,8 & 81 & 13,0 & 124 & 19,6 & 123 & 19,2 \\
\hline Atacama & 38 & 12,5 & 46 & 14,9 & 25 & 8,0 & 82 & 25,9 & 74 & 23,1 \\
\hline Coquimbo & 106 & 14,2 & 123 & 16,2 & 114 & 14,8 & 133 & 17,0 & 113 & 14,2 \\
\hline Valparaíso & 225 & 12,6 & 225 & 12,4 & 264 & 14,5 & 288 & 15,6 & 267 & 14,4 \\
\hline Metropolitana & 1238 & 17,3 & 1.470 & 20,3 & 1.427 & 19,5 & 1.390 & 18,8 & 1.754 & 23,4 \\
\hline O’Higgins & 66 & 7,3 & 86 & 9,4 & 86 & 9,4 & 56 & 6,0 & 63 & 6,7 \\
\hline Maule & 118 & 11,5 & 85 & 8,2 & 77 & 7,4 & 107 & 10,2 & 91 & 8,6 \\
\hline Bío-Bío & 200 & 9,6 & 204 & 9,7 & 251 & 11,9 & 250 & 11,7 & 274 & 12,8 \\
\hline Araucanía & 171 & 17,5 & 152 & 15,5 & 85 & 8,6 & 123 & 12,3 & 131 & 13,1 \\
\hline Los Ríos & 30 & 7,5 & 14 & 3,5 & 41 & 10,1 & 43 & 10,6 & 72 & 17,6 \\
\hline Los Lagos & 122 & 14,7 & 88 & 10,5 & 117 & 13,9 & 126 & 14,9 & 72 & 8,4 \\
\hline Aisén & 12 & 11,3 & 11 & 10,2 & 13 & 12,0 & 10 & 9,1 & 18 & 16,3 \\
\hline Magallanes & 14 & 8,6 & 32 & 19,5 & 30 & 18,2 & 31 & 18,7 & 26 & 15,6 \\
\hline Chile & 2.570 & 14,6 & 2.740 & 15,4 & 2.784 & 15,5 & 2.992 & 16,4 & 3.290 & 17,9 \\
\hline Sin dato & 3 & & 8 & & 25 & & 1 & & 1 & \\
\hline
\end{tabular}

cien mil habs. El resto de las regiones muestran riesgos similares o por debajo de lo nacional (Tabla 4).

Las muertes por Sida desde 1990 hasta el año 2015 fueron $9.850,87 \%$ de ellas correspondió a hombres, con una razón de 5,9 hombres por cada mujer. La mortalidad experimentó una disminución pasando de 3,5 por cien mil habs. en el año 2001 a 2,9 por cien mil habs. en el año 2015 (Tabla 5). Según distribución geográfica, en los últimos cinco años, la mortalidad se concentró en Arica-Parinacota y Tarapacá; estas regiones presentaron una tasa de 5,9 y 4,2 fallecidos por cien mil habs., respectivamente, en el año 2015. Se presentó un aumento de la mortalidad por Sida en la Región de Atacama el año 2015 (n = 19 casos, tasa 6,1 por cien mil habs.), el que duplica la tasa del año anterior y ocupa el primer lugar nacional ese año.

\section{Gonorrea}

Desde el año 2000, se observa una tendencia progresiva a la disminución de las tasas de gonorrea y una estabilización a partir del año 2011. Sin embargo, en el año 2015 se produce un aumento sostenido hasta el año 2017 (Tabla 6). El año 2017 se presentaron 2.768 casos, lo que representa $80 \%$ superior con respecto a la mediana del quinquenio anterior (1.533 casos). Según edad, el mayor riesgo se encuentra en el grupo de 15 a 24 años, destacándose que, a partir del año 2013, se desplaza paulatinamente la infección a edades menores. Es así que la tasa del grupo de 15 a 19 años superó al grupo de 25 a 29 años y siguió en aumento hasta el año 2017 alcanzando una tasa de 45,0 casos por cien mil habs. (Tabla 7). Entre el 2013 y el 2017, los hombres mostraron las mayores tasas, prevaleciendo en todo el período sobre las mujeres (Tabla 8). Ellos representaron $186 \%$ del total de casos, con una relación de 6 hombres por cada mujer en el quinquenio.

Con relación a la clasificación del tipo de gonorrea, en el quinquenio 2013-2017, 54\% fue notificado como infección gonocócica no especificada (A54.9) y 47\% se notificó como infección gonocócica del tracto urinario (A54.0-A54.2). La infección gonocócica del recto y ano representaron $0,4 \% ; 0,8 \%$ correspondió a faringitis gonocócica y la oftalmía gonocócica fue de 0,2\%. El $71 \%$ de los casos de gonorrea oftálmica se notificó en recién nacidos.

En el quinquenio 2013-2017, 4,7\% del total de casos de gonorrea se notificó en personas extranjeras, siendo $81 \%$ de sexo masculino. El año 2017 el porcentaje de extranjeros notificados representó $6,6 \%$ del total de casos. 


\begin{tabular}{|c|c|c|c|c|c|c|c|}
\hline \multirow[t]{2}{*}{ Año } & \multicolumn{2}{|c|}{ Ambos sexos } & \multicolumn{2}{|c|}{ Hombres } & \multicolumn{2}{|c|}{ Mujeres } & \multirow{2}{*}{$\begin{array}{c}\text { Razón } \\
\mathrm{H}: \mathrm{M}\end{array}$} \\
\hline & n Def. & Tasa* & n Def. & Tasa* & n Def. & Tasa* & \\
\hline 1990 & 72 & 0,5 & 66 & 1,0 & 6 & 0,1 & 11 \\
\hline 1991 & 77 & 0,6 & 75 & 1,1 & 2 & 0,0 & 37,5 \\
\hline 1992 & 114 & 0,8 & 101 & 1,5 & 13 & 0,2 & 7.8 \\
\hline 1993 & 174 & 1,3 & 163 & 2,4 & 11 & 0,2 & 14,8 \\
\hline 1994 & 253 & 1,8 & 227 & 3,2 & 26 & 0,4 & 8,7 \\
\hline 1995 & 302 & 2,1 & 278 & 3,9 & 24 & 0,3 & 11,6 \\
\hline 1996 & 362 & 2,5 & 317 & 4,4 & 45 & 0,6 & 7,0 \\
\hline 1997 & 410 & 2,8 & 360 & 4,9 & 50 & 0,7 & 7,2 \\
\hline 1998 & 383 & 2,6 & 343 & 4,6 & 40 & 0,5 & 8,6 \\
\hline 1999 & 474 & 3,1 & 424 & 5,6 & 50 & 0,7 & 8,5 \\
\hline 2000 & 458 & 3,0 & 402 & 5,3 & 56 & 0,7 & 7,2 \\
\hline 2001 & 552 & 3,5 & 479 & 6,2 & 73 & 0,9 & 6,6 \\
\hline 2002 & 440 & 2,8 & 380 & 4,9 & 60 & 0,8 & 6,3 \\
\hline 2003 & 423 & 2,7 & 357 & 4,6 & 66 & 0,8 & 5,4 \\
\hline 2004 & 399 & 2,5 & 356 & 4,5 & 43 & 0,5 & 8,3 \\
\hline 2005 & 390 & 2,4 & 331 & 4,1 & 59 & 0,7 & 5,4 \\
\hline 2006 & 422 & 2,6 & 357 & 4,4 & 65 & 0,8 & 5,5 \\
\hline 2007 & 398 & 2,4 & 347 & 4,2 & 51 & 0,6 & 6,8 \\
\hline 2008 & 392 & 2,3 & 328 & 4,0 & 64 & 0,8 & 5,1 \\
\hline 2009 & 435 & 2,6 & 365 & 4,4 & 70 & 0,8 & 5,2 \\
\hline 2010 & 435 & 2,5 & 370 & 4,4 & 65 & 0,8 & 5,7 \\
\hline 2011 & 472 & 2,7 & 386 & 4,5 & 86 & 1,0 & 4,5 \\
\hline 2012 & 456 & 2,6 & 378 & 4,4 & 78 & 0,9 & 4,8 \\
\hline 2013 & 523 & 3,0 & 456 & 5,2 & 67 & 0,8 & 6,8 \\
\hline 2014 & 506 & 2,8 & 424 & 4,8 & 82 & 0,9 & 5,2 \\
\hline 2015 & 528 & 2,9 & 451 & 5,1 & 77 & 0,8 & 5,9 \\
\hline Total & 9.850 & & 8.521 & & 1.329 & & \\
\hline
\end{tabular}

\section{Tabla 6. Casos y tasa de gonorrea. Chile 2000-2017*}

\begin{tabular}{rrr} 
Años & Casos & Tasas \\
2000 & 2.488 & 16,2 \\
2001 & 2.385 & 13,8 \\
2002 & 2.014 & 12,8 \\
2003 & 1.918 & 12,1 \\
2004 & 1.718 & 10,7 \\
2005 & 1.720 & 10,7 \\
2006 & 1.293 & 8,0 \\
2007 & 1.251 & 7,6 \\
2008 & 1.028 & 6,2 \\
2009 & 1.065 & 6,3 \\
2010 & 1.290 & 7,6 \\
\hline 2011 & 1.428 & 8,3 \\
2012 & 1.470 & 8,4 \\
2013 & 1.533 & 8,7 \\
\hline 2014 & 1.473 & 8,3 \\
\hline $2015^{*}$ & 1.797 & 10,0 \\
\hline $2016^{*}$ & 2.042 & 11,2 \\
\hline $2017^{*}$ & 2.768 & 15,1
\end{tabular}

*2015, 2016 y 2017 datos provisorios. Tasas por cien mil habs. Fuente: Base de datos ENO DEIS y Dpto. Epidemiología, DIPLAS, MINSAL, Chile.
Del total de notificaciones en el quinquenio, $0,4 \%$ de los casos declaró pertenecer a un pueblo originario.

Según distribución geográfica, el año 2017 los mayores riesgos se presentan en las regiones del extremo norte y sur del país en orden decreciente: Arica-Parinacota, Tarapacá, Antofagasta Los Lagos, Aysén y Coquimbo; el resto de las regiones se encuentran bajo la tasa nacional (Tabla 9).

\section{Sifilis}

A partir del año 2012 hasta el 2016, se observó una estabilización de las tasas de sífilis, entre 22 y 24 casos por cien mil habs. El año 2017 se produce un aumento de las notificaciones en $41 \%$ con relación al año anterior, notificándose 5.961 casos con una tasa de 32,4 por cien mil habs. (Tabla 10).

En el quinquenio 2013-2017, el grupo de 15 a 49 años representó 79\% del total de casos de sífilis, concentrándose el mayor riesgo entre los 20 a 39 años, quienes presentan las mayores tasas nacionales en los últimos dos años. El mayor aumento de las tasas el año 2017 se 


\begin{tabular}{|c|c|c|c|c|c|c|c|c|c|c|}
\hline \multirow[t]{2}{*}{ Grupo de edad } & \multicolumn{2}{|c|}{2013} & \multicolumn{2}{|c|}{2014} & \multicolumn{2}{|c|}{$2015^{*}$} & \multicolumn{2}{|c|}{$2016^{*}$} & \multicolumn{2}{|c|}{$2017^{*}$} \\
\hline & Casos & Tasas & Casos & Tasas & Casos & Tasas & Casos & Tasas & Casos & Tasas \\
\hline 0 - 4 años & 7 & 0,6 & 9 & 0,7 & 7 & 0,6 & 13 & 1,1 & 12 & 1,0 \\
\hline $5-9$ años & 6 & 0,5 & 3 & 0,2 & 10 & 0,8 & 6 & 0,5 & 9 & 0,7 \\
\hline $10-14$ años & 14 & 1,1 & 9 & 0,7 & 9 & 0,7 & 16 & 1,3 & 22 & 1,9 \\
\hline $15-19$ años & 301 & 22,0 & 305 & 22,7 & 365 & 27,6 & 474 & 36,5 & 576 & 45,0 \\
\hline $20-24$ años & 461 & 30,9 & 428 & 28,9 & 534 & 36,6 & 598 & 41,6 & 891 & 63,0 \\
\hline 25 - 29 años & 273 & 18,9 & 265 & 18,0 & 336 & 22,4 & 405 & 26,7 & 548 & 35,9 \\
\hline 30 - 34 años & 158 & 12,3 & 145 & 11,0 & 187 & 13,8 & 198 & 14,2 & 264 & 18,4 \\
\hline 35 - 39 años & 88 & 7,1 & 87 & 7,0 & 108 & 8,7 & 111 & 8,8 & 138 & 10,8 \\
\hline 40 - 44 años & 64 & 5,1 & 63 & 5,1 & 72 & 5,8 & 73 & 5,9 & 106 & 8,5 \\
\hline 45 - 49 años & 49 & 3,9 & 54 & 4,3 & 55 & 4,4 & 54 & 4,3 & 60 & 4,8 \\
\hline 50 - 54 años & 41 & 3,5 & 52 & 4,3 & 46 & 3,8 & 40 & 3,2 & 56 & 4,5 \\
\hline 55 - 59 años & 30 & 3,1 & 27 & 2,7 & 32 & 3,0 & 28 & 2,6 & 35 & 3,1 \\
\hline 60 - 64 años & 23 & 3,0 & 14 & 1,8 & 18 & 2,2 & 10 & 1,2 & 24 & 2,7 \\
\hline 65 - 69 años & 10 & 1,7 & 3 & 0,5 & 5 & 0,8 & 10 & 1,5 & 12 & 1,7 \\
\hline 70 - 74 años & 3 & 0,7 & 5 & 1,1 & 7 & 1,4 & 2 & 0,4 & 9 & 1,7 \\
\hline $75-79$ años & 3 & 0,9 & 1 & 0,3 & 3 & 0,9 & 1 & 0,3 & 5 & 1,4 \\
\hline 80 y más años & 2 & 0,6 & 3 & 0,8 & 2 & 0,5 & 3 & 0,7 & 0 & 0,0 \\
\hline Total & 1.533 & 8,7 & 1.473 & 8,3 & 1.796 & 10,0 & 2.042 & 11,2 & 2.767 & 15,1 \\
\hline
\end{tabular}

Tabla 8. Casos y tasas de gonorrea según sexo. Chile 2013-2017*

\begin{tabular}{lccccc}
\hline Años & \multicolumn{2}{c}{ Hombres } & Mujeres & Relación Hombre:Mujer \\
& Casos & Tasas & Casos & Tasas & 6,4 \\
\hline 2013 & 1.327 & 15,2 & 206 & 2,3 & 6,8 \\
\hline 2014 & 1.285 & 14,6 & 188 & 2,1 & 6,5 \\
\hline $2015^{*}$ & 1.556 & 17,5 & 240 & 2,6 & 5,8 \\
\hline $2016^{*}$ & 1.740 & 19,3 & 302 & 3,3 & 5,3 \\
\hline
\end{tabular}

*2015, 2016 y 2017 datos provisorios (año 2015 y 20171 casos sin sexo). Tasas por cien mil habs. Fuente: Depto. Epidemiología, DEIS. DIPLAS - Ministerio de Salud de Chile.

agrupó en estas edades (entre 61 y 79 casos por cien mil habs.) (Tabla 11).

Según sexo, la tasa de hombres supera a la de mujeres a partir del año 2008. El año 2017, ellos representan 65\% del total de casos, con una relación de 1,9 hombres por cada mujer (Tabla 12). A partir de los 20 años se observa un predominio del sexo masculino. El año 2017 en el grupo de adolescentes (15 a 19 años) las tasas en ambos sexos son similares. Según etapa, del total de casos $54 \%$ se notifica como sífilis precoz y $27 \%$ como sífilis tardía.

Las mujeres gestantes con sífilis representan $21 \%$ del total de casos notificados en mujeres (426 casos) y la sífilis congénita presenta una tasa de 0,04 por mil nacidos vivos el año 2017. 


\begin{tabular}{|c|c|c|c|c|c|c|c|c|c|c|}
\hline \multirow[t]{2}{*}{ Regiones } & \multicolumn{2}{|c|}{2013} & \multicolumn{2}{|c|}{2014} & \multicolumn{2}{|c|}{$2015^{*}$} & \multicolumn{2}{|c|}{$2016^{*}$} & \multicolumn{2}{|c|}{$2017^{*}$} \\
\hline & Casos & Tasas & Casos & Tasas & Casos & Tasas & Casos & Tasas & Casos & Tasas \\
\hline Arica & 50 & 21,6 & 35 & 14,9 & 41 & 17,1 & 66 & 27,1 & 143 & 57,9 \\
\hline Tarapacá & 116 & 36,2 & 177 & 53,8 & 181 & 53,7 & 206 & 59,8 & 203 & 57,6 \\
\hline Antofagasta & 102 & 16,9 & 117 & 19,1 & 198 & 31,8 & 218 & 34,5 & 338 & 52,7 \\
\hline Atacama & 23 & 7,6 & 15 & 4,9 & 37 & 11,8 & 27 & 8,5 & 24 & 7,5 \\
\hline Coquimbo & 93 & 12,4 & 93 & 12,2 & 139 & 18,0 & 86 & 11,0 & 144 & 18,1 \\
\hline Valparaíso & 176 & 9,8 & 124 & 6,9 & 128 & 7,0 & 126 & 6,8 & 160 & 8,6 \\
\hline Metropolitana & 453 & 6,3 & 396 & 5,5 & 440 & 6,0 & 511 & 6,9 & 818 & 10,9 \\
\hline O'Higgins & 42 & 4,7 & 36 & 4,0 & 38 & 4,1 & 46 & 5,0 & 39 & 4,2 \\
\hline Maule & 29 & 2,8 & 41 & 4,0 & 63 & 6,0 & 58 & 5,5 & 79 & 7,5 \\
\hline Biobío & 109 & 5,2 & 125 & 6,0 & 164 & 7,8 & 167 & 7,8 & 312 & 14,6 \\
\hline Araucanía & 57 & 5,8 & 35 & 3,6 & 63 & 6,4 & 102 & 10,2 & 81 & 8,1 \\
\hline Los Ríos & 25 & 6,3 & 27 & 6,7 & 25 & 6,2 & 26 & 6,4 & 58 & 14,1 \\
\hline Los Lagos & 197 & 23,8 & 197 & 23,6 & 252 & 30,0 & 344 & 40,6 & 340 & 39,8 \\
\hline Aysén & 51 & 48,0 & 51 & 47,5 & 23 & 21,2 & 45 & 41,2 & 26 & 23,6 \\
\hline Magallanes & 10 & 6,1 & 4 & 2,4 & 5 & 3,0 & 14 & 8,5 & 3 & 1,8 \\
\hline Total & 1.533 & 8,7 & 1.473 & 8,3 & 1.797 & 10,0 & 2.042 & 11,2 & 2.768 & 15,1 \\
\hline
\end{tabular}

\begin{tabular}{|ccc|}
\hline Tabla 10. Casos y tasas de sífilis. Chile $\mathbf{2 0 0 0 - 2 0 1 7}{ }^{*}$ & \\
\hline Años & Casos & Tasas \\
\hline 2000 & 3.680 & 23,9 \\
\hline 2001 & 3.206 & 20,6 \\
\hline 2002 & 2.792 & 22,2 \\
\hline 2003 & 2.982 & 22,5 \\
\hline 2004 & 3.173 & 19,7 \\
\hline 2005 & 2.818 & 17,4 \\
\hline 2006 & 2.971 & 18,2 \\
\hline 2007 & 3.013 & 17,9 \\
\hline 2008 & 3.239 & 19,4 \\
\hline 2009 & 3.376 & 20,0 \\
\hline 2010 & 3.374 & 19,8 \\
\hline 2011 & 3.939 & 22,8 \\
\hline 2012 & 4.340 & 24,9 \\
\hline 2013 & 4.355 & 24,7 \\
\hline 2014 & 4.411 & 24,8 \\
\hline $2015^{*}$ & 4.159 & 23,1 \\
\hline $2016^{*}$ & 4.188 & 23,0 \\
\hline $2017 *$ & 5.961 & 32,4 \\
\hline *2015, 2016 y 2017 datos provisorios. Tasas por cien mil habs. Fuente: Base de datos ENO. DEIS. \\
\hline Dpto. Epidemiología, MINSAL, Chile. & & \\
\hline & & \\
\hline
\end{tabular}

En el quinquenio 2013-2017, 4,4\% del total de casos de sífilis se notificaron en personas extranjeras, siendo $58 \%$ de sexo masculino. El año 2017, el porcentaje de extranjeros notificados representó 7,5\% del total. En este mismo quinquenio, $0,3 \%$ de los casos declaró pertenecer a un pueblo originario.

Según distribución geográfica, en los últimos dos años, las tasas se mantuvieron altas en Tarapacá, Antofagasta, Coquimbo, Valparaíso y Región Metropolitana, todas ellas superando la tasa nacional. Se destaca el aumento de la tasa en Arica-Parinacota el año 2017 (Tabla 13).

\section{Hepatitis $B$}

A partir del año 2008, se observa una tendencia sostenida al alza en las tasas de notificación, esto es producto de una notificación retroactiva. La mayor tasa se presenta en el año 2013 (8,2 por cien mil habs.), para luego descender y estabilizarse los últimos tres años. El año 2017 la tasa fue de 6,0 por cien mil habs. (Tabla 14).

El año 2017 el grupo de 20 a 39 años concentró 64\% del total de los casos y presentó las mayores tasas (entre 9,7 y 14, 2 por cien mil habs.) (Tabla 15). La mediana de edad en el último quinquenio fue de 34 años.

Los hombres representaron $83 \%$ del total de los casos ( 5 hombres por cada mujer) y sus tasas superan a aquellas en mujeres en todo el periodo. (Tabla 16). 


\begin{tabular}{|c|c|c|c|c|c|c|c|c|c|c|}
\hline \multirow[t]{2}{*}{ Grupos de edad } & \multicolumn{2}{|c|}{2013} & \multicolumn{2}{|c|}{2014} & \multicolumn{2}{|c|}{$2015^{*}$} & \multicolumn{2}{|c|}{$2016^{*}$} & \multicolumn{2}{|c|}{$2017^{*}$} \\
\hline & Casos & Tasa & Casos & Tasa & Casos & Tasa & Casos & Tasa & Casos & Tasa \\
\hline 0 - 4 años & 37 & 3,0 & 29 & 2,3 & 33 & 2,7 & 27 & 2,2 & 23 & 1,9 \\
\hline $5-9$ años & 1 & 0,1 & 1 & 0,1 & 0 & 0,0 & 0 & 0,0 & 2 & 0,2 \\
\hline 10 - 14 años & 11 & 0,9 & 10 & 0,8 & 7 & 0,6 & 4 & 0,3 & 9 & 0,8 \\
\hline $15-19$ años & 258 & 18,8 & 238 & 17,7 & 244 & 18,4 & 255 & 19,6 & 334 & 26,2 \\
\hline 20 - 24 años & 729 & 48,8 & 668 & 45,1 & 676 & 46,3 & 683 & 47,5 & 979 & 69,4 \\
\hline $25-29$ años & 671 & 46,6 & 675 & 45,8 & 710 & 47,4 & 730 & 48,1 & 1.132 & 74,4 \\
\hline 30 - 34 años & 587 & 45,7 & 539 & 40,8 & 553 & 40,7 & 603 & 43,2 & 882 & 61,6 \\
\hline 35 - 39 años & 455 & 36,8 & 456 & 36,8 & 390 & 31,3 & 443 & 35,3 & 677 & 53,2 \\
\hline 40 - 44 años & 391 & 31,4 & 379 & 30,4 & 358 & 28,8 & 344 & 27,7 & 471 & 37,9 \\
\hline 45 - 49 años & 313 & 24 & 364 & 28.9 & 295 & 23,4 & 304 & 24,2 & 404 & 32,4 \\
\hline 50 - 54 años & 263 & 22,4 & 283 & 23,6 & 232 & 19,0 & 244 & 19,7 & 296 & 23,8 \\
\hline 55 - 59 años & 185 & 19,2 & 212 & 21,0 & 189 & 18,0 & 176 & 16,1 & 233 & 20,8 \\
\hline 60 - 64 años & 118 & 15,6 & 152 & 19,3 & 141 & 17,1 & 96 & 11,2 & 127 & 14,2 \\
\hline 65 - 69 años & 104 & 17,3 & 132 & 21,3 & 101 & 15,7 & 92 & 13.8 & 137 & 19,8 \\
\hline 70 - 74 años & 70 & 15,6 & 108 & 23,1 & 91 & 18,7 & 72 & 14,2 & 105 & 20,1 \\
\hline 75 - 79 años & 75 & 23,4 & 84 & 25,3 & 59 & 17,2 & 51 & 14,4 & 78 & 21,2 \\
\hline 80 y más años & 85 & 23,9 & 78 & 21,1 & 80 & 20,8 & 64 & 16,0 & 71 & 17,0 \\
\hline Total & 4.353 & 24,7 & 4.408 & 24,7 & 4.159 & 23,1 & 4.188 & 23,0 & 5.960 & 32,5 \\
\hline
\end{tabular}

Tabla 12. Casos y tasas de sífilis según sexo. Chile 2013-2017*

\begin{tabular}{lllllc}
\hline Años & Casos & Hombres & Mujeres & Relación Hombre:Mujer \\
& 2.311 & 26,5 & 2.042 & 22,9 & 1,1 \\
\hline 2013 & 2.348 & 26,6 & 2.060 & 22,9 & 1,1 \\
\hline 2014 & 2.246 & 25,2 & 1.913 & 21,0 & 1,2 \\
\hline $2016^{*}$ & 2.650 & 29,4 & 1.538 & 16,7 & 1,7 \\
\hline $2017^{*}$ & 3.892 & 42,8 & 2.068 & 22,3 & 1,9 \\
\hline
\end{tabular}

*2015, 2016 y 2017 datos provisorios. Tasas por cien mil habs. (Año 2013, 2014 y 2017 un caso sin edad). Fuente: Base de datos ENO. DEIS. Dpto. Epidemiología, MINSAL, Chile.

En el año 2017, el porcentaje de notificaciones en etapa aguda representa $70 \%$ del total de la hepatitis. En términos de tasas, la hepatitis aguda concentra 4,2 casos por cien mil habs. y la forma crónica 1,8 por cien mil habs.

Las mujeres gestantes con hepatitis B representaron $11 \%$ del total de casos notificados en mujeres (21 casos) el año 2017. Este mismo año no se notificaron niños por esta infección.

En el quinquenio 2013-2017, 5,3\% del total de casos de hepatitis B se notificaron en personas extranjeras, siendo 69\% de sexo masculino. El año 2017 el porcentaje de extranjeros notificados representó $10 \%$ del total. En el mismo quinquenio, $0,2 \%$ de los casos declaró pertenecer a un pueblo originario. 


\begin{tabular}{|c|c|c|c|c|c|c|c|c|c|c|}
\hline \multirow[t]{2}{*}{ Regiones } & \multicolumn{2}{|c|}{2013} & \multicolumn{2}{|c|}{2014} & \multicolumn{2}{|c|}{$2015^{*}$} & \multicolumn{2}{|c|}{$2016^{*}$} & \multicolumn{2}{|c|}{$2017^{*}$} \\
\hline & Casos & Tasas & Casos & Tasas & Casos & Tasas & Casos & Tasas & Casos & Tasas \\
\hline Arica & 61 & 26,4 & 53 & 22,5 & 50 & 20,9 & 43 & 17,7 & 88 & 35,6 \\
\hline Tarapacá & 184 & 57,3 & 184 & 56,0 & 143 & 42,5 & 150 & 43,5 & 204 & 57,8 \\
\hline Antofagasta & 181 & 30,0 & 174 & 28,4 & 192 & 30,8 & 182 & 28,8 & 262 & 40,9 \\
\hline Atacama & 67 & 22,0 & 75 & 24,3 & 46 & 14,7 & 68 & 21,5 & 83 & 25,9 \\
\hline Coquimbo & 151 & 20,2 & 162 & 21,3 & 208 & 27,0 & 181 & 23,1 & 275 & 34,7 \\
\hline Valparaíso & 753 & 42,1 & 726 & 40,1 & 615 & 33,7 & 568 & 30,8 & 589 & 31,7 \\
\hline Metropolitana & 1.690 & 23,7 & 1.772 & 24,5 & 1.658 & 22,7 & 1.841 & 24,9 & 2939 & 39,3 \\
\hline O'Higgins & 276 & 30,6 & 246 & 27,0 & 254 & 27,6 & 188 & 20,3 & 230 & 24,6 \\
\hline Maule & 126 & 12,3 & 156 & 15,1 & 111 & 10,6 & 99 & 9,4 & 125 & 11,8 \\
\hline Biobío & 458 & 21,9 & 433 & 20,6 & 475 & 22,5 & 444 & 20,9 & 641 & 30,0 \\
\hline Araucanía & 116 & 11,9 & 68 & 6,9 & 80 & 8,1 & 104 & 10,4 & 203 & 20,3 \\
\hline Los Ríos & 15 & 3,8 & 52 & 12,9 & 48 & 11,9 & 57 & 14,0 & 82 & 20,0 \\
\hline Los Lagos & 242 & 29,2 & 210 & 25,2 & 217 & 25,8 & 219 & 25,8 & 204 & 24,8 \\
\hline Aysén & 12 & 11,3 & 16 & 14,9 & 12 & 11,1 & 8 & 7,3 & 12 & 10,9 \\
\hline Magallanes & 23 & 14,1 & 84 & 51,3 & 50 & 30,4 & 36 & 21,7 & 28 & 16,8 \\
\hline Total & 4.355 & 24,7 & 4.411 & 24,8 & 4.159 & 23,1 & 4.188 & 23,0 & 5.961 & 32,5 \\
\hline
\end{tabular}

\begin{tabular}{|c|c|c|}
\hline Años & Casos & Tasas \\
\hline 1997 & 149 & 1,0 \\
\hline 1998 & 162 & 1,1 \\
\hline 1999 & 172 & 1,1 \\
\hline 2000 & 224 & 1,5 \\
\hline 2001 & 271 & 1,8 \\
\hline 2002 & 230 & 1,5 \\
\hline 2003 & 224 & 1,4 \\
\hline 2004 & 247 & 1,5 \\
\hline 2005 & 195 & 1,2 \\
\hline 2006 & 263 & 1,6 \\
\hline 2007 & 297 & 1,8 \\
\hline 2008 & 361 & 2,2 \\
\hline 2009 & 508 & 3,0 \\
\hline 2010 & 1.103 & 6,5 \\
\hline 2011 & 1.080 & 6,3 \\
\hline 2012 & 1.059 & 6,1 \\
\hline 2013 & 1.437 & 8,2 \\
\hline 2014 & 1.206 & 6,8 \\
\hline 2015 & 1.030 & 5,7 \\
\hline 2016 & 1.115 & 6,1 \\
\hline $2017^{*}$ & 1.103 & 6,0 \\
\hline
\end{tabular}

Según distribución geográfica, en los últimos dos años, las tasas de hepatitis B se mantuvieron altas en el extremo norte: Tarapacá, Antofagasta, Atacama y en la Región Metropolitana, todas ellas superando la tasa nacional (Tabla 17).

\section{Conclusiones}

Según resultado de la vigilancia, sífilis es la ITS de mayor tasa de notificación, la que, a su vez, experimentó un aumento el año 2017. Le sigue la infección por VIH. En términos generales, todas las ITS presentan una relativa estabilización de sus tasas en el período 2014 y 2015 , excepto gonorrea que mostró un aumento en estos años, la que se focalizó en el grupo de 15 a 24 años. No, obstante, el año 2017 sífilis e infección por VIH presentaron un incremento de sus tasas en relación al año 2016.

En todas estas infecciones, el riesgo de los hombres supera al de las mujeres y el grupo de edad más afectado es el de 15 a 39 años. Según distribución geográfica las regiones de Arica-Parinacota a Antofagasta, Metropolitana, Valparaíso, Los Lagos y Aysén, presentan los mayores riesgos.

El comportamiento ascendente de la gonorrea en población de 15 a 24 años, es un potente indicador de que existe un aumento de otras infecciones de transmisión sexual (ITS) en ellos (VIH, sífilis, hepatitis B entre otras), considerando que comparten el mismo mecanismo de 


\begin{tabular}{|c|c|c|c|c|c|c|c|c|c|c|}
\hline \multirow[t]{2}{*}{ Grupos de edad } & \multicolumn{2}{|c|}{2013} & \multicolumn{2}{|c|}{2014} & \multicolumn{2}{|c|}{$2015^{*}$} & \multicolumn{2}{|c|}{$2016 *$} & \multicolumn{2}{|c|}{$2017^{*}$} \\
\hline & Casos & Tasa & Casos & Tasa & Casos & Tasa & Casos & Tasa & Casos & Tasa \\
\hline 0 - 4 años & 2 & 0,2 & 0 & 0,0 & 0 & 0,0 & 1 & 0,1 & 0 & 0,0 \\
\hline 5 - 9 años & 0 & 0,0 & 0 & 0,0 & 1 & 0,1 & 0 & 0,0 & 1 & 0,1 \\
\hline $10-14$ años & 1 & 0,1 & 3 & 0,2 & 3 & 0,2 & 1 & 0,1 & 3 & 0,3 \\
\hline $15-19$ años & 58 & 4,2 & 52 & 3,9 & 34 & 2,6 & 48 & 3,6 & 69 & 5,4 \\
\hline 20 - 24 años & 213 & 14,3 & 177 & 12,0 & 161 & 11,0 & 177 & 12,4 & 193 & 13,7 \\
\hline $25-29$ años & 215 & 14,9 & 195 & 13,2 & 190 & 12,7 & 221 & 14,7 & 216 & 14,2 \\
\hline 30 - 34 años & 222 & 17,3 & 185 & 14,0 & 165 & 12,2 & 170 & 12,1 & 169 & 11,8 \\
\hline 35 - 39 años & 164 & 13,3 & 155 & 12,5 & 103 & 8,3 & 107 & 8,6 & 124 & 9,7 \\
\hline 40 - 44 años & 136 & 10,9 & 111 & 8,9 & 82 & 6,6 & 102 & 8,2 & 70 & 5,6 \\
\hline 45 - 49 años & 131 & 10,4 & 99 & 7,8 & 65 & 5,2 & 80 & 6,4 & 59 & 4,7 \\
\hline 50 - 54 años & 99 & 8,4 & 70 & 5,8 & 70 & 5,7 & 66 & 5,3 & 63 & 5,1 \\
\hline 55 - 59 años & 74 & 7,7 & 50 & 5,0 & 65 & 6,2 & 62 & 5,7 & 42 & 3,7 \\
\hline 60 - 64 años & 50 & 6,6 & 31 & 3,9 & 42 & 5,1 & 37 & 4,2 & 31 & 3,5 \\
\hline 65 - 69 años & 32 & 5,3 & 34 & 5,5 & 28 & 4,4 & 25 & 3,8 & 33 & 4,8 \\
\hline 70 - 74 años & 24 & 5,4 & 32 & 6,9 & 9 & 1,8 & 10 & 2,0 & 18 & 3,4 \\
\hline 75 - 79 años & 12 & 3,7 & 9 & 2,7 & 6 & 1,7 & 3 & 0,8 & 7 & 1,9 \\
\hline 80 y más años & 3 & 0,8 & 2 & 0,5 & 7 & 1,8 & 2 & 0,5 & 5 & 1,2 \\
\hline Total & 1.436 & 8,2 & 1.205 & 6,8 & 1.031 & 5,7 & 1.112 & 6,1 & 1.103 & 6,4 \\
\hline
\end{tabular}

\begin{tabular}{|c|c|c|c|c|c|}
\hline \multirow[t]{2}{*}{ Años } & \multicolumn{2}{|c|}{ Hombres } & \multicolumn{2}{|c|}{ Mujeres } & \multirow[t]{2}{*}{ Relación Hombre:Mujer } \\
\hline & Casos & Tasas & Casos & Tasas & \\
\hline 2013 & 1.257 & 14,4 & 179 & 2,1 & 7,0 \\
\hline 2014 & 1.039 & 11,8 & 166 & 2,0 & 6,3 \\
\hline 2015 & 910 & 10,2 & 121 & 1,3 & 7,5 \\
\hline $2016^{*}$ & 970 & 10,8 & 142 & 1,6 & 6,8 \\
\hline $2017^{*}$ & 917 & 10,1 & 186 & 2,0 & 4,9 \\
\hline
\end{tabular}

*Datos provisorios. Tasas por cien mil habs. (Año 2013 y 2014 un caso sin edad). Fuente: Base de datos ENO.DEIS. Dpto. Epidemiología, MINSAL, Chile.

transmisión. Pero, a diferencia de gonorrea que presenta un corto período de incubación, las otras ITS se caracterizan por presentar manifestaciones clínicas a largo plazo y, por tanto, no se diagnostican al momento de la infección.

Según los resultados de la vigilancia de ITS el año 2017, existe una tasa ascendente de gonorrea en la población de 15 a 29 años, y a su vez un incremento de las tasas de sífilis e infección por VIH. Considerando que las co-infecciones de transmisión sexual aumentan la capacidad de transmisión del $\mathrm{VIH}^{4}$, que la menor edad está fuertemente asociada con múltiples ITS $^{5}$ y que existe un bajo uso de condón en nuestro país ${ }^{6}$, es necesario generar acciones específicas y que impacten, permitiendo asentar conductas de sexo seguro y consulta precoz ante el riesgo 


\begin{tabular}{|c|c|c|c|c|c|c|c|c|c|c|}
\hline \multirow[t]{2}{*}{ Regiones } & \multicolumn{2}{|c|}{2013} & \multicolumn{2}{|c|}{2014} & \multicolumn{2}{|c|}{2015} & \multicolumn{2}{|c|}{$2016^{*}$} & \multicolumn{2}{|c|}{$2017^{*}$} \\
\hline & Casos & Tasas & Casos & Tasas & Casos & Tasas & Casos & Tasas & Casos & Tasas \\
\hline Arica & 24 & 10,4 & 19 & 8,1 & 19 & 7,9 & 14 & 5,8 & 11 & 4,5 \\
\hline Tarapacá & 33 & 10,3 & 37 & 11,3 & 35 & 10,4 & 45 & 13,1 & 30 & 8,5 \\
\hline Antofagasta & 69 & 11,4 & 50 & 8,2 & 51 & 8,2 & 67 & 10,6 & 69 & 10,8 \\
\hline Atacama & 15 & 4,9 & 14 & 4,5 & 9 & 2,9 & 12 & 3,8 & 24 & 7,5 \\
\hline Coquimbo & 33 & 4,4 & 26 & 3,4 & 45 & 5,8 & 41 & 5,2 & 32 & 4,0 \\
\hline Valparaíso & 129 & 7,2 & 73 & 4,0 & 74 & 4,1 & 102 & 5,5 & 116 & 6,2 \\
\hline Metropolitana & 845 & 11,8 & 785 & 10,9 & 614 & 8,4 & 556 & 7.5 & 531 & 7,1 \\
\hline O'Higgins & 26 & 2,9 & 37 & 4,1 & 36 & 3,9 & 49 & 5,3 & 52 & 5,6 \\
\hline Maule & 31 & 3,0 & 17 & 1,6 & 25 & 2,4 & 44 & 4,2 & 38 & 3,6 \\
\hline Biobío & 110 & 5,3 & 89 & 4,2 & 77 & 3,6 & 127 & 6,0 & 116 & 5,4 \\
\hline Araucanía & 23 & 2,4 & 16 & 1,6 & 12 & 1,2 & 19 & 1,9 & 18 & 1,8 \\
\hline Los Ríos & 3 & 0,8 & 10 & 2,5 & 6 & 1,5 & 4 & 1,0 & 11 & 2,7 \\
\hline Los Lagos & 54 & 6,5 & 20 & 2,4 & 17 & 2,0 & 25 & 2,9 & 42 & 4,9 \\
\hline Aysén & 3 & 2,8 & 4 & 3,7 & 5 & 4,6 & 2 & 1.8 & 2 & 1,8 \\
\hline Magallanes & 39 & 24,0 & 9 & 5,5 & 6 & 3,6 & 5 & 3,0 & 11 & 6,6 \\
\hline Total & 1.437 & 8,2 & 1.206 & 6,8 & 1.031 & 5,7 & 1.112 & 6,1 & 1.103 & 6,0 \\
\hline
\end{tabular}

de infección, para así, asegurar el tratamiento oportuno y el control de la transmisión de la infección.

En relación al aumento sostenido de gonorrea, el Ministerio de Salud se encuentra estableciendo y ejecutando un plan de medidas específicas de control, prevención y vigilancia dirigido a la red pública y privada de salud.

En este escenario epidemiológico, además, es fundamental mantener activa y reforzar periódicamente la importancia de la vigilancia epidemiológica de forma oportuna y con calidad en todos los niveles involucrados, con el objetivo de contar con información veraz que permita la toma de decisiones en salud pública.

Se debe tener presente que las ITS en menores de edad (si se descarta la transmisión de madre a hijo por vía vertical) debe alertar al equipo de salud, ya que puede ser producto de abuso o violación sexual, el que debe ser denunciado según se establece en Ley № 19.696 Código procesal penal, Ley $\mathrm{N}^{\mathrm{o}} 20.207$ delitos sexuales contra menores, Ley $N^{o} 19.617$ delito de violación y la Guía Clínica de Abuso Sexual (MINSAL).

Como poblaciones más vulnerables se incluye a las personas pertenecientes a pueblos originarios y a los migrantes. Si bien, según este análisis, estas poblaciones no presentan grandes porcentajes de infecciones, existe un aumento en la población migrante en el año 2017, lo que no implica que el aumento de algunas ITS este dado por el incremento en la población extranjera. Además, cabe considerar que esta población presenta barreras de acceso a la atención de salud, particularmente en derecho de salud sexual y reproductiva y una menor adscripción al sistema de salud.

\section{Resumen}

Las infecciones de transmisión sexual (ITS) son una de las principales causas de enfermedad aguda, infertilidad, discapacidad a largo plazo y muerte en el mundo ${ }^{1}$. Este informe presenta la situación epidemiológica preliminar de las ITS hasta el año 2017 en Chile. Sífilis es la ITS de mayor tasa de notificación, seguido de la infección por VIH. En términos generales, todas las ITS presentan una relativa estabilización de sus tasas en el período 2014 y 2015 , excepto gonorrea que muestra un incremento en estos años, el que se focaliza en el grupo de 15 a 24 años. El año 2017, sífilis y la infección por VIH presentaron un aumento de sus tasas en relación al año 2016. En todas estas ITS la tasa de hombres supera a la de mujeres y el grupo de edad más afectado es el de 15 a 39 años. Según distribución geográfica, las regiones de Arica-Parinacota a Antofagasta, Metropolitana, Valparaíso, Los Lagos y Aysén, presentan los mayores riesgos. 


\section{Documentos recomendados}

Se encuentran disponibles en el sitio web del Ministerio de Salud, la siguiente normativa, para vigilancia, manejo y control:

- Circular B51/24 del 23/05/2013. "Vigilancia epidemiológica de sífilis y gonorrea". Disponible en: http://epi.minsal.cl/wp-content/uploads/2016/05/ CIRCULAR_24_SIFILIS_GONORREA_2013.pdf

- Circular B51/35 del 14/10/2010. Vigilancia epidemiológica del VIH/SIDA. Disponible en: http://epi. minsal.cl/wp-content/uploads/2016/02/CircularVIHSIDAnew.pdf

- Circular B51/16 del 30/03/2009. "Vigilancia epidemiológica de sífilis y medidas de control de la hepatitis B y la hepatitis C". Disponible en: http://epi.minsal.cl/ wp-content/uploads/2015/11/CircularHep_ByC.pdf

- "Norma de manejo y tratamiento de las infecciones de transmisión sexual. Disponible en: http://epi.minsal.cl/ wp-content/uploads/2016/07/Norma-103-Infeccionesde-Transmisi\%C3\%B3n-Sexual-1.pdf

- Decreto 206 del 2005. Reglamento sobre infecciones de transmisión sexual. Disponible en: http://epi.minsal. cl/wp-content/uploads/2016/07/REGLAMENTO206-Infecciones-de-Transmision-Sexual-1.pdf

- Norma Conjunta de Prevención de la Transmisión Vertical del VIH y la Sífilis. Disponible en: http:// diprece.minsal.cl/wrdprss minsal/wp-content/ uploads/2015/01/NORMA-DE-PTV-VIH-SIFILIS. pdf

- Orientaciones para los procesos claves en las enfermedades transmisibles por sangre: infección por virus hepatitis B, hepatitis C, HTLV I, enfermedad de Chagas y sífilis. Disponible en: http://web.minsal.cl/ sites/default/files/files/OrientacionesparaprocesosclavesETporsangre2011.pdf

- Otros documentos sobre ITS disponibles en: http:// diprece.minsal.cl/programas-de-salud/programa-vihsida-e-its/informacion-al-profesional-vih-sida-e-its/

- Otros documentos sobre hepatitis B disponibles en: http://diprece.minsal.cl/programas-de-salud/programas-enfermedades-transmisibles/hepatitis-b-y-c/

\section{Agradecimientos}

A todas las personas que son parte de la red epidemiológica de vigilancia de las ITS, del Instituto de Salud Pública y de los Programas de ITS en las SEREMIS, Servicios de Salud, establecimientos de salud públicos y privados y laboratorios quienes construyen y fortalecen esta vigilancia.

Informe elaborado por: Karen Cáceres Burton.

\section{Referencias bibliográficas}

1.- Organización Panamericana de la Salud (OPS); Organización Mundial de la Salud (OMS); Programa Conjunto de las Naciones Unidas sobre el VIH/Sida (ONUSIDA).

Pautas Vigilancia de las Infecciones de Transmisión Sexual.1999 Disponible en: http://www.who.int/hiv/pub/sti/en/cds_csr_ edc_99_3sp.pdf.

2.- Organización Mundial de la Salud (OMS). Estrategia mundial de prevención y control de las infecciones de transmisión sexual: 2006 2015: romper la cadena de transmisión.
Disponible en: http://apps.who.int/iris/ bitstream/10665/43773/1/9789243563473_spa. pdf.

3.- Ministerio de Salud de Chile. Decreto 158: Reglamento sobre notificación de enfermedades transmisibles de declaración obligatoria. 22/10/2004. Disponible en: http://epi.minsal.cl/ wp-content/uploads/2016/03/DECRETO158_ editado.pdf.

4.- Kalichman SC, Pellowski J, Turner C. Prevalence of sexually transmitted coinfections in people living with HIV/AIDS: systematic review with implications for using HIV treatments for prevention. Sex Trans
Infect 2011; 87 (3): 183-90. doi: 10.1136/ sti.2010.047514.

5.- Feldblum PJ. Lie Ch, Weaver MA, Van Damme L, Halpern V, Adeiga A, et al. Baseline factors associated with incident HIV and STI in four microbicide trials. Sex Trans Dis 2010; 37 (10): 594-601 DOI: $10.1097 /$ OLQ.0b013e3181e15f0b.

6.- Dpto. de Epidemiología, DIPLAS_MINSAL Encuesta Calidad de Vida y Salud 20152016. Disponible en: http://epi.minsal.cl/ wp-content/uploads/2017/06/Resultados Abril2017_ENCAVI_2015-16_Depto_ Epidemiolog\%C3\%ADDa_MINSAL.pdf. 